\title{
TOWARD FEATURE ALGEBRAS IN VISUAL DATABASES: THE CASE FOR A HISTOGRAM ALGEBRA
}

\author{
Amarnath Gupta \\ San Diego Supercomputer Center \\ La Jolla, CA \\ gupta@sdsc.edu
}

\section{Simone Santini}

University of California, San Diego

La Jolla, CA

ssantini@ece.ucsd.edu

\begin{abstract}
Searching and managing large archives of visual data, such as images and video, is made hard by the lack of proper integration between the visual aspects of the problem (image processing, motion estimation, feature extraction...) and its database aspects (defining visual data as data types in a database). In this paper, we argue that image database languages can be built based on feature algebras, and demonstrate how such a feature algebra can be built in the case of one of the most popular image characterization techniques: histograms.
\end{abstract}

Keywords: Histograms, Databases, Image Features, Algebras, Content Based Image Retrieval

\section{INTRODUCTION}

A primary capability of any database system is to provide a user the means to create, query and manipulate data in a natural, meaningful and expressive way. In traditional database systems (Abiteboul et al., 1995), this is accomplished by using well-formed mathematical structures (such as sets or trees), and designing a language to create, constrain and manipulate data sets associated with these structures. The language, based on an algebra or calculus, is designed to express most queries that a user is likely to formulate on the mathematical structure for the 
domains of application. The implicit assumption behind the choice of the mathematical structure and the operators of the language is that they fit naturally to the way a user would think about the data.

Unfortunately, in query and data manipulation languages designed for visual (image and video) database systems, a balance between meaningfulness and expressive power has been hard to achieve. We believe that one primary reason for this difference is that the visual scientist's focus is often targeted toward problem-specific data transformation, while the database scientist's focus is on generic methods to formalize relationships between data, and to access data from well-structured collections. This difference in focus, we contend, has led to an "expressiveness gap" in database languages for visual information systems. We illustrate the problem in the following paragraphs.

Consider a hypothetical database system created by assimilating "best practices" from current visual database research. Let us assume that the system supports both image and video retrieval.

We also start with the assumption that each image or video item is identified by an $i d$ and Id denotes the type of all identifiers in the system. Being a characteristic system, it will typically have a set of global visual features $\left\{g_{i}\right\}$, computed by a series of image transforms, reducing the original image to a collection of numbers, the so-called feature vectors (Gupta, 1995). These feature vectors represent different perceptual or cognitive properties of an image such as color, texture, or camera motion properties of videos, and can be designed using sophisticated techniques to attain properties like invariance to affine transformations and illumination disparities. However, despite the fact that each feature vector is a collection, the user does not usually have access to any value "inside" this collection, and has to treat the collection as the instance of an opaque data type $\mathbf{T}_{i}^{g}$. In fact, most often the user does not even have access to the value of the feature. The type has only one binary operation $\circ_{i}: \mathbf{T}_{i}^{g} \times \mathbf{T}_{i}^{g} \rightarrow \mathbf{R}$, producing a distance between two instances of type $\mathbf{T}_{i}^{g}$. Depending on $\mathbf{T}_{i}^{g}$, ${ }_{i}$ may be commutative (e.g., when $\circ_{i}$ is a norm such as $L_{2}$ ), but it need not be (Santini and Jain, 1999), and the associativity of the operator is not considered important. Since the distance operation produces a real number called score, the database system often compares one example image with others in the database and produces $[\mathbf{I d} \times \mathbf{R}]$, a rank-ordered list of $\{i d$, score $\}$ pairs as the result type of a search.

As the system supports $n$ independent global image features, we can assume without loss of generality that these features form a relation. So the collection of images and their properties can be viewed as a relational 
database with tuples having the type $\mathbf{T}_{1}^{g} \times \mathbf{T}_{2}^{g} \ldots \mathbf{T}_{n}^{g} \times \mathbf{I d}$. The system offers a composite distance between two images by computing a tuplewise difference. This tuple uses a combination function-(Fagin, 1999) calls it a scoring rule-over the tuple of $\left\{i d\right.$, score $_{i}$ pairs obtained from each of the $n$ features. Thanks to the wide body of recent research on rules and formal techniques to express and compute this combination function (Fagin and Wimmers, 1999; Fagin, 1999; Adali et al., 1998; Nepal and Ramakrishna, 1999) our hypothetical system will have a rich collection of ways to use aggregate ranking functions.

Thus, given the lack of access to the value or the structure of a feature, the system treats individual features as a "black box" with very poor support. However, it provides a wide variety of utilities when the features are put in a relation and when the tuple distance function produces a list of scores.

The situation improves somewhat for local visual features. A local visual feature type $\mathbf{T}_{i}^{l}$ is defined for an image as a composite type $\mathbf{T}_{i}^{f} \times$ $\mathbf{R}^{2}$, where the first component arises from image transformations and the second component localizes the feature in a region of the image. For local video feature, the composite type is given by $\mathbf{T}_{i}^{f} \times \mathbf{R}^{2} \times \tau$, where $\tau$ stands for the time when the feature occurs in the video ${ }^{1}$. We call these locality components the spatial support and the temporal support of the feature respectively. Given the composite nature of local visual features, the "support" component of the feature can be projected out. This allows our hypothetical system to characterize the spatial support region into known spatial data types, such as regions, lines and points. Now, the system can provide a rich set of query operations defined on spatial and temporal intervals and on binary topological relations that can be derived from spatial support data. ( $\mathrm{Li}$ et al., 1997; Li et al., 1998; Day et al., 1998). Again we have the same problem that the feature part of the data is significantly weak and unexpressive compared to the structured portion of the data.

\subsection{CONTRIBUTION OF THE PAPER}

In this paper, we attempt to show that it is possible to reduce this expressiveness gap by defining mathematical structures for several feature classes in a data and process independent fashion. To this end, we first identify a set of properties that such feature algebra will need to satisfy. Then we develop a special case of feature algebra by treating histograms as a generic mathematical structure. We illustrate that this allows us to perform manipulations and express query classes on visual data that could not be expressed unless a feature algebra were defined. 


\section{SOME PROPERTIES OF A FEATURE ALGEBRA}

We classify features into global, local and relational. A local feature is characterized either by an explicit spatial or temporal support, or by an implicit encoding of spatial (or temporal) information in a globally computed feature (e.g., color correlograms (Huang et al., 1997). Relational features are often represented as graphs having atomic attributes or computed features at the nodes and edges. In this section we discuss the structural representation of a generic feature that may be global, the non-support component of a local property, or embedded in the node of a relational attribute.

Definition 2.1 $A$ feature is a collection $C$ of values, containing arbitrarily nested subcollections $S(C)$ indexed by an algebraic structure $\mathbf{M}$ defined over $\mathbf{N}$, the set of natural numbers.

Example 1. Structurally, a vector feature $V$ can be considered to be a one-dimensional array, and defined as a set of real values, indexed by a list of natural numbers, such that $V[i]$ is the $i$-th element of $V$.

Example 2. Many complex features such as texture are often represented by a hierarchical bank of filters applied to an image. For example, (Hatipoglu et al., 1999) describes a dual-tree complex wavelet feature tree for texture determination. In this case the $S_{i}(C)$ is formed by the coefficients of each individual filter bank $C=\cup_{i} S_{i}(C)$. M represents an extended tree-structure such that the node of the tree indexes a filter bank, and, a list within a node specifies a single coefficient within a filter bank.

While the operators in a specific feature algebra must depend on the exact nature of $C$ and $\mathbf{M}$, we can identify a core set of properties that most feature algebras will satisfy. One set of operators is governed by the domain of feature values (e.g., integer, real), and is outside the algebra itself. The algebra would include operators such as the following:

selectCollection. Chooses a specific collection from a database. This can be accomplished by a predicate on some attribute of the collection such as its name, or cardinality.

pickCollection. In case of collections containing subcollections, this operation selects a subcollection a path-expression from the "root" of the collection.

nextSubCollection. Applicable for nested collections, this operation relies on the premise that the structure $\mathbf{M}$ provides a traversal 
functionality. Thus, given a subcollection this operator selects the next subcollection in the traversal order prescribed by $\mathbf{M}$.

selectElement. This is a classical select operation of a database system.

pickElement. This is a selection by path expression starting from the root of a subcollection containing the element. Typically, the path expression will be prefixed by the path expression leading up to the subcollection.

nextElement. Similar to the nextSubCollection operation, this operator traverses from one element to the following element.

getElementValue. Given an element, this operator returns its value. In some cases this value may be complex. For example, for a 3D object it may return the two 3-vector principal curvature directions of a surface.

compareElement. This corresponds to the $\circ_{i}: \mathbf{T}_{i}^{g} \times \mathbf{T}_{i}^{g} \rightarrow \mathbf{R}$ mentioned before and produces an element distance.

makeCollection. The operation creates a new (sub)collection from zero or more existing (sub)collections, by possibly applying a function $f$ to them. An example of such a function could be creating a new histogram by computing a termwise difference of two given histograms.

placeSubCollection. This operation positions a newly constructed subcollection into a specific point in the structure of a larger collection. It is based on the premise that the structure $\mathbf{M}$ allows a systematic traverse and insert functionality.

From this generic set of operations we now illustrate a concrete instance of a feature algebra, applied to the case of histograms.

\section{THE HISTOGRAM ALGEBRA}

A histogram is a frequency distribution of one or more variables over a set of observations. Without loss of generality, we may state that given any measurement function $f: R \rightarrow \Re^{m}$, where $R$ is the domain of definition of an image (usually $R \subset \Re^{2}$ ) a histogram represents the probability distribution of the values of $f$. The goal of our algebra is to preserve this probabilistic character of the histogram, instead of treating them like an array (Libkin et al., 1997; Marathe and Salem, 1997), where no correlation between different cells of an array can be assumed. 
Before describing the histogram data type and the operations on it, we will need a few accessory functions and definitions. The data types boolean, integer, real, as well as arrays of these types are assumed. Integers can be used to form ranges, such as $1: n$. If $a$ is an array, $a[i]$ (or, indifferently, $a_{i}$ ) is its $i$ th element, and $a[1: n]$ (or $\left.a_{1: n}\right)$ is an array composed of its first $n$ components. Ranges can be $k$-dimensional, and a range can be assigned to variables of type range, but no other operations are defined on them. The $k$-dimensional range $\left\{d_{1 i} \leq x_{i} \leq d_{2 i}, i=1, \ldots k\right\}$ will be represented using the notation $\left[d_{1 i}, d_{2 i}\right]^{k}$ where $d_{1 i}$ and $d_{2 i}$ are one-dimensional arrays.

A probability distribution function is a mapping from real numbers to the interval $(0,1)$ obeying the laws of probability.

The generation function $g_{p}\left(\left[d_{1 i}, d_{2 i}\right]^{k}, n^{k}, p_{i}\right)$ generates $\mathrm{n}$ real numbers in the $\mathrm{k}$-dimensional range $\left[d_{1 i}, d_{2 i}\right]^{k}$ according to the probability distribution function given by $p_{i}$. The number of buckets in each dimension is specified by $n^{k} \in N^{k}$.

A bucketing function $b_{n, D, \Delta}: \mathbf{R}^{k} \rightarrow[1: n]^{k}$, where $D$ is a range $\left[d_{1 i}, d_{2 i}\right]^{k}$, and $\Delta$ is a $k$-dimensional array of positive numbers, is a function that maps the $k$-dimensional range $\left[d_{1 i}, d_{2 i}\right]^{k}$ into the integer range $[1: n]^{k}$. An element of this range is called a bucket. The semantics of the bucketing function is as follow. Let $\mathbf{X}$ be a k-dimensional array, and $x_{i} \in \mathbf{X}$ such that

$$
\left\lfloor\frac{x_{i}-d_{1 i}}{\Delta_{i}}\right\rfloor+1=h_{i}
$$

then

$$
b_{n, D, \Delta}(\mathbf{X})=\left[h_{1}, h_{2}, \ldots, h_{k}\right]
$$

Definition 3.1 A histogram is a mapping $H:\left[1: n_{i}\right]^{k} \rightarrow \mathbf{R}^{m} \cup$ null, where $k$ is the dimension of the histogram, $n_{i}$ is the bucket size along the $i$-th dimension of the histogram, and $m$ is the codomain dimension of the histogram.

While by definition the codomain of a histogram is always 1 , if two distinct histograms $H 1$ and $H 2$ have exactly the same dimensions, domain, and bucket sizes, we represent them in a compressed histogram with a codomain 2 . To see where such a histogram may be used, consider a 2D edge-orientation histogram with the two dimensions representing the direction of the orientation and the strength of the edge respectively. We may now want to perform a smoothing operation along each of the dimensions, thereby computing at each cell two values. We represent the $k$-th value in the $i j$-th cell of the composite $2 \mathrm{D}$ histogram $H^{\prime}$ as $H^{\prime}[i][j][k]$ as if it had an additional dimension. For a simple (non-composite) 2D histogram, $H^{\prime}[i][j][k]$ would produce the value err. 
An important decision in our algebra is that we strictly enforce dimensionality of histograms. In linear algebra it is quite common to identify a column vector with a matrix with only one column, or a row vector with a matrix with only one row. For that matter, it is possible to see a vector as an array of any dimensionality in which all dimensions but one have only one element. The same identification is possible with histograms: a single dimensional histogram can be seen as a two (or three, or four...)-dimensional histogram with only one bucket in the second direction. This identification is explicitly prohibited in our model. The dimensionality of a histogram is a well-defined attribute irrespective of the number of buckets along any dimension. Two histograms are called isomorphic iff they have the same dimensionality, codomain dimensionality, bucket dimensionality along every dimension, and if their domains coincide.

Constructors. The following operators build histograms starting from other data types.

Let $\mathbf{A}$ be a $k$-dimensional array. The operator build $(\mathbf{A})$ constructs a $k$-dimensional histogram with codomain dimension equal 1 such that $H\left(i_{1}, \ldots, i_{n}\right)=\mathbf{A}\left[i_{1}, \ldots, i_{n}\right]$. The operator build $\left(v, i_{1}, \ldots, i_{k}\right)$ builds a $k$ dimensional histogram with co-domain dimension equal to the dimension of the array $\mathrm{v}$, and $i_{i}$ buckets along the $i$ th dimension. The histogram is initialized to the map

$$
H\left(j_{1}, \ldots, j_{k}\right)= \begin{cases}v & \text { if } j_{1}=i_{1} \wedge j_{2}=i_{2} \wedge \cdots \wedge j_{k}=i_{k} \\ 0 & \text { otherwise }\end{cases}
$$

We will also consider two special operators. The first, $G_{p}$, constructs a histogram based on a given function. Its semantics is

$$
G_{p}\left(\left[d_{1}, d_{2}\right]^{k}, f, n^{k}\right)=\operatorname{build}\left(g_{p}\left(\left[d_{1}, d_{2}\right]^{k}, n^{k}, f\right)\right)
$$

The operator $\operatorname{null}\left(n_{1}, \ldots, n_{k}\right)$ builds a $k$-dimensional histogram with $n_{i}$ buckets along the $i$ th dimension implementing the null mapping.

Histogram functions. The following functions compute quantities related to an histogram without altering their argument.

$\operatorname{dim}(H)$ returns the dimension of the histogram.

$\operatorname{dom}_{i}(H)$ returns the domain of the histogram in the i-th dimension.

$\operatorname{Dom}(H)$ is a macro that returns the complete domain of the histogram. size $_{i}(H)$ returns the number of buckets in the histogram for the $i$-th dimension. 
$\operatorname{Size}(H)$ is a macro that returns an array $\left[n_{1}, \ldots, n_{k}\right]$ denoting the size of all buckets in the histogram.

$\operatorname{val}\left(H\left(i_{1}, \ldots, i_{k}\right)\right)$ returns the value of the bucket at the specified index. bounds $\left(H\left(i_{1}, \ldots i_{k}\right)\right)$ returns a k-tuple of pairs $\left[\left[u_{1}, l_{1}\right], \ldots\left[u_{k}, l_{k}\right]\right]$ where $\left[u_{j}, l_{j}\right]$ are the upper and lower bounds on the domain of the bucket $\left[i_{1}, \ldots, i_{k}\right]$ for the $j$-th dimension.

For conveneience, we also define the predicate $e q\left(H\left[i_{1}, \ldots, i_{k}\right]=\right.$ const $)$, which evaluates to true iff $\operatorname{val}\left(H\left[i_{1}, \ldots, i_{k}\right]\right)=$ const, and the macro $\operatorname{Tot} \operatorname{Count}(H)$ which computes the sum of $\operatorname{val}\left(H\left[i_{1}, \ldots, i_{k}\right]\right)$ over all buckets.

Same size operators. The following operators combine two histograms of the same dimension, codomain dimension and bucket size, or operate on a histogram without altering its structure. The operators are undefined when applied to histograms that differ on any of these dimensions.

The macro norm $(H)$ normalizes a histogram so that, if $G=\operatorname{norm}(H)$

$$
\sum_{i_{1}, \ldots, i_{k}} H\left(i_{1}, \ldots i_{k}\right)=1
$$

and, for all the values for which the operation is defined

$$
\frac{G\left(i_{1}, \ldots, i_{k}\right)}{G\left(j_{1}, \ldots, j_{k}\right)}=\frac{H\left(i_{1}, \ldots, i_{k}\right)}{H\left(j_{1}, \ldots, j_{k}\right)}
$$

The operator + denotes addition of two histograms. $H_{1}+H_{2}$ has the following semantics:

$$
\left(H_{1}+H_{2}\right)\left(i_{1}, \ldots, i_{k}\right)=H_{1}\left(i_{1}, \ldots, i_{k}\right)+H_{2}\left(i_{1}, \ldots, i_{k}\right)
$$

The operator - denotes absolute value difference of two histograms. $H_{1}-H_{2}$ has the following semantics:

$$
\left(H_{1}-H_{2}\right)\left(i_{1}, \ldots, i_{k}\right)=\left|H_{1}\left(i_{1}, \ldots, i_{k}\right)-H_{2}\left(i_{1}, \ldots, i_{k}\right)\right|
$$

These operators are special cases of the general element-wise combination operators $\langle\cdot\rangle$ defined as follows. Let $f: \mathbf{R}^{m} \times \mathbf{R}^{m} \rightarrow\left(\mathbf{R}^{+}\right)^{m}$ be a symmetric and associative operator. Then, for histograms $H_{1}$ and $H_{2}$ with $m$-dimensional co-domain, $H_{1}\langle f\rangle H_{2}$ has the following semantics:

$$
\left(H_{1}\langle f\rangle H_{2}\right)\left(i_{1}, \ldots, i_{k}\right)=f\left(H_{1}\left(i_{1}, \ldots, i_{k}\right), H_{2}\left(i_{1}, \ldots, i_{k}\right)\right)
$$

The operator $=$ denotes assignment and has the usual semantics:

$$
H 1[i 1, \ldots, i k]=C \Leftrightarrow e q(H 1[i 1, \ldots, i k]=C)=\text { true }
$$

where $C$ is a constant. 
Field operators. These operators combine a histogram and a real number. The unary operator - denotes scalar multiplication by a positive scalar constant. The operator is not defined for $c<0$. Thus $c \cdot H$ (abbreviated $c H)$ has the semantics:

$$
(c \cdot H)\left(i_{1}, \ldots, i_{k}\right)=c H\left(i_{1}, \ldots, i_{k}\right)
$$

Similarly to the previous case, this operator is a special case of the general operator $\langle\cdot\rangle$, defined as follows. Let $f: \mathbf{R} \times \mathbf{R}^{m} \rightarrow\left(\mathbf{R}^{+}\right)^{m}$ a function, then the semantics of $c\langle f\rangle H$ is

$$
(c\langle f\rangle H)\left(i_{1}, \ldots, i_{k}\right)=f\left(c, H\left(i_{1}, \ldots, i_{k}\right)\right)
$$

Cross Dimensional Operators.

These operators change the dimensionality or the number of buckets of a histogram. The expand operator increases the dimensionality of a histogram. Its signature is $G=\operatorname{expand}(H, n, a)$, where $H$ is a $k$-dimensional histogram, $a$ is a $k$ dimensional vector of integers such that $a_{i} \in[1, n]$ and $i \neq j \Rightarrow a_{i} \neq a_{j}$, and the result is an $n$-dimensional histogram.

The formal specification of the operator (see below) is rather involved, but its semantics is actually quite simple. For example, consider a two dimensional histogram $H$. Then the operation $G=\operatorname{expand}(H, 3,[3,1])$ builds a three dimensional histogram such that the first dimension of $H$ becomes the third dimension of $G$, the second dimension of $H$ becomes the first dimension of $G$, and all other dimensions of $G$ (in this case the second dimension) have only one bin. In this case, the relation between $G$ and $H$ is:

$$
\begin{aligned}
\operatorname{size}_{1}(G) & =\operatorname{size}_{2}(H) \\
\operatorname{size}_{2}(G) & =1 \\
\operatorname{size}_{3}(G) & =\operatorname{size}_{1}(H) \\
G(i, 1, j) & =H(j, i)
\end{aligned}
$$

In order to define the behavior of the operator in more general circumstances, we need a few auxiliary definitions. Let $I=\left[i_{1}, \ldots i_{k}\right] \in N^{k}$ be a $k$-dimensional index, and $J=\left[j_{1}, \ldots, j_{n}\right] \in N^{n}$ an $n$-dimensional index. Let $u_{a}: N^{k} \rightarrow N^{n}$ be the transformation defined as

$$
\left[u_{a}(J)\right]_{i}= \begin{cases}j_{h} & \text { if } a_{h}=i \\ 1 & \text { otherwise }\end{cases}
$$

Consider now the set of $n$-indices that have a " 1 " in the locations not covered by the vector $a: \mathcal{I}_{a}=\left\{\left[j_{1}, \ldots, j_{n}\right] \in N^{n} \mid \nexists h: a_{h}=q \Rightarrow j_{q}=1\right\}$. Then the transformation $u_{a}$ is an isomorphism between $N^{k}$ and $\mathcal{I}_{a}$ and 
therefore invertible on this domain: $u^{-1}: \mathcal{I}_{a} \rightarrow N^{k}$. The relation between the histograms $H$ and $G$ can then be defined as follows:

$$
\forall I \in \mathcal{I}_{a} \quad G(I)=H\left(u_{a}^{-1}(I)\right)
$$

The embed operator substitutes part of a larger histogram with values from a smaller histogram with the same dimensionality, starting at a location $I=\left[i_{1}, \ldots, i_{n}\right]$ in the larger histogram. The operator signature is $Q=\varepsilon_{I}(H, G)$, where $H$ is embedded into $G$. If for some $j$ we have $i_{j} \leq 0$ or $i_{j}+\operatorname{size}_{j}(H)>\operatorname{size}_{j}(G)$, the histogram $H$ will be clipped, and only the values with indices within the legal range of $G$ will be used. The semantics of the operator is

$$
\left(\varepsilon_{I}(H, G)\right)(J)= \begin{cases}H\left(\left[j_{1}-i_{1}, \ldots, j_{n}-j_{n}\right]\right) & \text { if } \forall h 0<j_{h}-i_{h} \leq s i z e_{h}(H) \\ G\left(\left[j_{1}, \ldots, j_{n}\right]\right) & \text { otherwise }\end{cases}
$$

where $J=\left[j_{1}, \ldots, j_{n}\right]$.

The select operator $\sigma_{\theta}(H)$, selects those bins of histogram $H$, that satisfy the predicate $\theta$. Its semantics is given by:

$$
\sigma_{\theta}(H)= \begin{cases}H[i] & \text { if } \theta(H[i])=\text { true } \\ \text { null } & \text { otherwise }\end{cases}
$$

The project operator $\pi_{h, \oplus}(H)$ takes a symmetric associative operator $\oplus: \mathbf{R}^{m} \times \mathbf{R}^{m} \rightarrow\left(\mathbf{R}^{+}\right)^{m}$ and uses it to compress the $h$ th dimension of the histogram $H$. The semantics of the operator is the following

$$
\left(\pi_{h, \oplus}(H)\right)\left(i_{1}, \ldots, i_{h-1}, i_{h+1}, \ldots, i_{n}\right)=\bigoplus_{j=1}^{\text {size }_{h}(H)} H\left(i_{1}, \ldots, i_{h-1}, j, i_{h+1}, \ldots, i_{n}\right)
$$

Next, the traversal operator $T_{f}(H)$ takes as an argument an index transform $f: N^{k} \rightarrow N^{n}(n \geq k)$ and uses it to reduce the dimensionality of the histogram $H$ and transform its indices

$$
\left(T_{f}(H)\right)(I)=H(f(I))
$$

where $I \in N^{k}$ is an index of the new histogram. As an example, consider the two dimensional histogram of Fig. 1 and the index transform defined by Table 1, corresponding to the traversal of Fig. 1.b. The traversed histogram is shown in Fig. 1.c.

Finally, the rebucket operator changes the number of buckets along all the dimensions of a histogram re-distributing the data inside a bucket according to a given probablity density. Consider, for instance, the onedimensional histogram in Fig. 2, The histogram has three buckets, and 


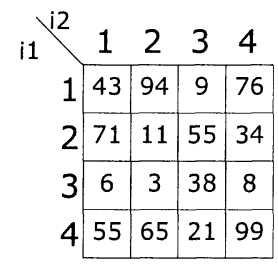

(a)

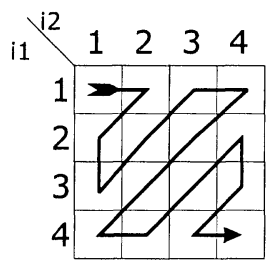

(b)

\begin{tabular}{|c|c|c|c|c|c|c|c|c|c|c|c|c|c|c|c|}
1 & 2 & 3 & 4 & 5 & 6 & 7 & 8 & 9 & 10 & 11 & 12 & 13 & 14 & 15 & 16 \\
\hline 43 & 94 & 71 & 6 & 11 & 9 & 76 & 55 & 3 & 55 & 65 & 38 & 34 & 8 & 21 & 99 \\
\hline
\end{tabular}

(c)

Figure 1 The traversal operator.

\begin{tabular}{|c|cccccccc|}
\hline$i$ & 1 & 2 & 3 & 4 & 5 & 6 & 7 & 8 \\
$f(i)$ & $(1,1)$ & $(1,2)$ & $(2,1)$ & $(3,1)$ & $(2,2)$ & $(1,3)$ & $(1,4)$ & $(2,3)$ \\
\hline$i$ & 9 & 10 & 11 & 12 & 13 & 14 & 15 & 16 \\
$f(i)$ & $(3,2)$ & $(1,4)$ & $(2,4)$ & $(3,3)$ & $(4,2)$ & $(4,3)$ & $(3,4)$ & $(4,4)$ \\
\hline
\end{tabular}

Table 1

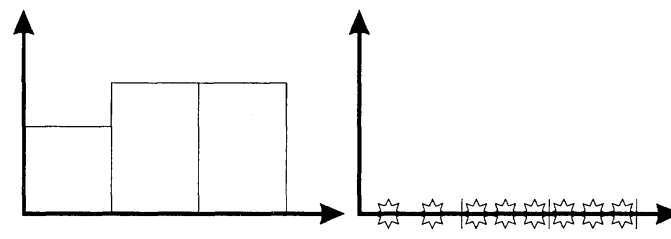

(a) (b)

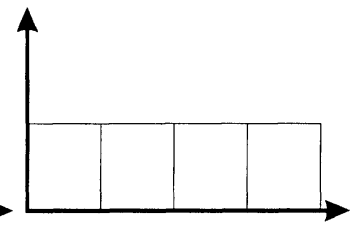

(c)

Figure 2 An example of re-bucketing

we want to expand it to four using a uniform underlying probability. The rebucket operator works as follows:

1 Transform the histogram into a statistical sampling using the existing buckets and the given probability. In other words: the histogram was obtained by sampling a probability distribution and, according to the result, there were two samples in the interval covered by the first bucket, three samples in the interval covered by the second bucket, and three samples in the interval covered by 
the third bucket. Inside the buckets, the samples are distributed according to the given distribution (uniformly, in this case).

2 The statistical distribution is re-sampled with the new number of buckets, four in this case.

The operator has the form $b(H, q, p)$, where $H$ is a $n$-dimensional histogram, $q \in N^{n}$ specifies the number of buckets in the final histogram, and $p$ is a probability density function $p: \mathcal{H} \times N^{n} \times \Re^{n} \rightarrow[0,1]$ that specifies the distribution of the samples inside a bucket. Note that $p$ can depend on the histogram and the index of the bucket that we are expanding.

\section{COMPUTING WITH THE HISTOGRAM ALGEBRA}

We now illustrate how the algebra can be used to compute useful operations.

Sum of squares of a histogram.

$$
s q(H)=S(H\langle\cdot\rangle H)
$$

where

$$
S(H)= \begin{cases}\pi_{1,+}(H) & \text { if } \operatorname{dim}(H)=1 \\ S\left(\pi_{1,+}(H)\right) & \text { otherwise }\end{cases}
$$

The recursion is well defined since for all histograms $H \operatorname{dim}\left(\pi_{h, \oplus}(H)\right)=$ $\operatorname{dim}(H)-1$.

Computation of the $L_{2}$ distance between histograms.

$$
L_{2}\left(H_{1}, H_{2}\right)=\sqrt{\mathrm{sq}\left(H_{1}-H_{2}\right)}
$$

Computation of the Hue histogram from the RGB histogram.

We assume that the functions $h(r, g, b), s(r, g, b) v(r, g, b)$ transform an $\mathrm{r}, \mathrm{g}, \mathrm{b}$ color into the corresponding hsv color. From this function, given a histogram with $n$ bins on each color axis, an index for the corresponding hsv color can be computed by the function

$$
\iota[i, j, k]=n \cdot\left[h\left(\frac{i}{n}, \frac{j}{n}, \frac{k}{n}\right), s\left(\frac{i}{n}, \frac{j}{n}, \frac{k}{n}\right), v\left(\frac{i}{n}, \frac{j}{n}, \frac{k}{n}\right)\right]
$$

The hues histgram can then be computed as

$$
\operatorname{hue}(H)=\pi_{3,+}\left(\pi_{2,+}\left(T_{\iota}(H)\right)\right)
$$


Answering queries.

The algebra provides a powerful tool for the specification of queries in interactive systems. Due to the greater complexity of these examples, we will assume that the histogram algebra is expressed in a suitable programming language. In particular, we will write the functions using the ML programming language (Ullman, 1997). The reasons why ML was chosen are its powerful handling of functions, including support for currying. In ML, a function $f$ that takes an integer and returns an integer (e.g. fun $f(x:$ int $)=x$ ) is a first class object of type int $\rightarrow$ int. On the other hand, a function defined as fun $x y$ $=\mathrm{x} * \mathrm{y}$, where all the variables are integers, is a curried function of type int $\rightarrow$ (int $\rightarrow$ int) that is, the function takes an integer value $(x)$ and produces a function that takes the value $y$ and returns an integer. In other words, the expression $f(x)$ is a function of type int $\rightarrow$ int.

The rest of the ML syntax used in the following is rather intuitive, and should make the examples understandable also to readers not familiar with ML.

Example 1. "Find all the histograms that behave like the function $f$ in the interval $[I, J]$ ". A similarity measure for this query is given by

$-\operatorname{fun} D(f, F, I, J)=$

let

hist1 $=\sigma_{I \leq x \leq J}\left(G_{p}(\operatorname{Dom}(\mathrm{H}), \mathrm{f}, \operatorname{Size}(\mathrm{H}))\right)$;

hist2 $=\sigma_{I \leq x \leq J}(\mathrm{H})$;

in

$L_{2}$ (hist1 - hist2)

end;

Example 2. "Find all peaks of a histogram". The peaks are to be determined using the following rules: first the histogram is filtered with a given kernel $K=\left[w_{-m}, \ldots, w_{0}, \ldots, w_{m}\right]$, then the maxima are detected. Points adjacents to a peak are not considered peaks. For the sake of simplicity, we will only consider one-dimensional histograms.

We begin with the definition of two functions that shift a histogram by an amount $I$, where $I$ is an index. The first function pads the shifted portion with zeros:

$\begin{aligned} &-\operatorname{fun} \operatorname{shft1}(\mathrm{H}, \mathrm{I})= \\ & \varepsilon_{I}(\operatorname{null}(\operatorname{Size}(\mathrm{H})), \mathrm{H})\end{aligned}$

The second function rotates the histogram, as if it were a periodic function 
- fun $\operatorname{shft2}(H, I)=$

$\varepsilon_{I}(\operatorname{null}(\operatorname{Size}(\mathrm{H})), \mathrm{H})+\varepsilon_{-\operatorname{ImodSize}(H)}(\operatorname{null}(\operatorname{Size}(\mathrm{H})), \mathrm{H})$.

The filter operator, with kernel $K$ is defined as follows:

- fun $F(K, m, H)=$

let

fun $F t(K, m, H)=$

if $\mathrm{m}=1$

$\mathrm{k}[1] * \mathrm{H}$

else

in

$(k[1] * H)+F t(k[2: m], m-1, \operatorname{shft2}(H, 1))$

$\mathrm{Ft}(\mathrm{K}, 2 * \mathrm{~m}+1, \operatorname{shft} 2(\mathrm{H},-\mathrm{m}))$

end;

Note that in this case we use the second shift operator that is, we consider the histogram as a periodic function. If this is undesired, the histogram can be embedded into a null histogram of size $\operatorname{Size}(H)+2 m$ and the first shift operator can be used without losing data.

The peaks will be returned in the form of a histogram that is zero everywhere except in the peak locations, where it has value 1 . We will use the indicator function $\delta(a, b)$ whose value is 1 if $a=b$ and zero otherwise. The following operator takes a histogram $H$ and returns a histogram with all bins set to zero except where $H$ attains its maximum:

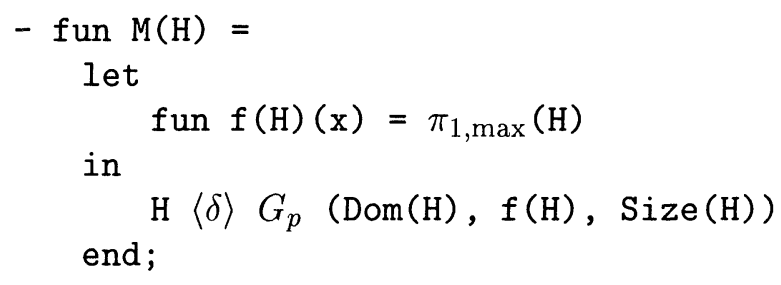

The following auxiliary function takes an histogram $H$ and a histogram $G$ with the same dimension and bucket dimension as $H$. For every bin in $G$ with a nonzero value creates a three bins in $H$ with zero value. In other words, the function creates "holes" in $H$ of size 3 corresponding to the values in $G$. 


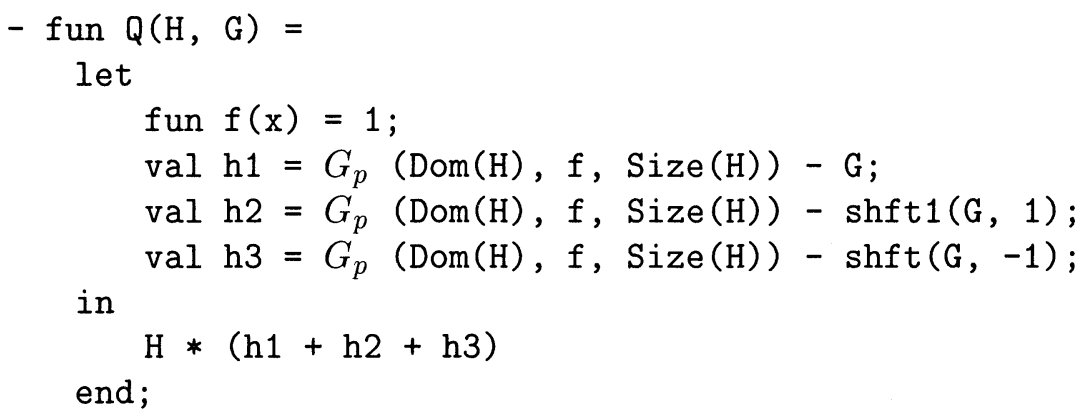

Finally, the function $P$, finds the $n$ highest peaks in the histogram

- fun $P(H, n, K, m)=$

let

fun $\operatorname{Pt}(H, n)=$

if $\mathrm{n}=1$

$\mathrm{M}(\mathrm{H})$

else

$$
M(H)+P(Q(H, M(H)), n-1)
$$

in

$\operatorname{Pt}(F(K, m, H), n)$

end;

Example 3. "Find whether, at a specific point in time $t$ of a video sequence, the dominant motion in the image is reversed (change of approximately 180 degrees) in less than three frames". We are looking for the event that the motion is reversed sometimes between $t$ and $t+3$.

The data are stored in a relational database whose schema comprises just one table:

$$
\operatorname{MOTION}(t: \text { int }, h: \text { histogram })
$$

where $t$ is the time at which the motion is considered, and $h$ is a histogram counting how many points are moving in a given direction.

We begin by writing a function that determines whether, given two histograms $H_{1}$ and $H_{2}$, there is an inversion of motion between the two. The function works as follows (see Fig. 3). We first take, for each histogram, the highest peak, which is representative of the dominant motion, then we add the two histograms to obtain a single histogram with two peaks. Using the function $\mathrm{P}$ defined above, we can write this histogram as

$$
P\left(H_{1}, 1\right)+P\left(H_{2}, 1\right)
$$

We then proceed as illustrated in Fig. 4. The histogram of the two 

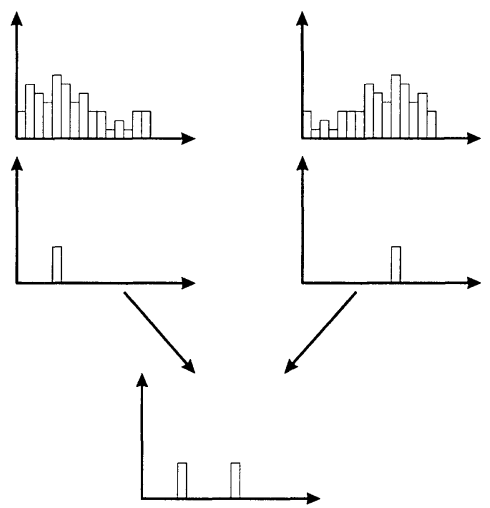

Figure 3 First steps of the determination of motion inversion between two histograms.

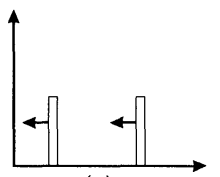

(a)

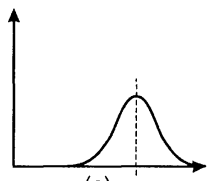

(c)

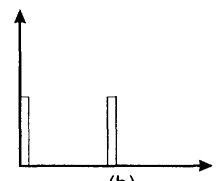

(b)

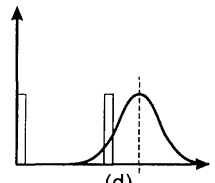

(d)

Figure 4 Determination of the distance of two peaks of a histogram

major peaks, represented in Fig. 4.a is shifted until the lowest peak is on the first bin (Fig. 4.b). Then, a "gauge" histogram is created using a Gaussian function centered at a bin corresponding to the distance at which we want to check the presence of a peak (the Gaussian allows us to tolerate slight misplacements of the second peak, and to control the extent to which these displacements can be accomodated), as in Fig. 4.c. Finally, the Gaussian histogram and the peak histogram are multiplied, giving us a measure of the presence of a second peak in the desired position. The shift function $S h$ uses the function $s h f t 2$ defined above: 
- fun $\operatorname{Sh}(H)=$

let

fun $f(x)=$ if $x=0$ then 1 else 0 ;

val $\operatorname{cond}=\operatorname{proj}(1, \max , H * G(\operatorname{Dom}(H), f, \operatorname{Size}(H))) !=0$; in

if cond then $\mathrm{H}$ else $\operatorname{Sh}(\operatorname{shft2}(\mathrm{H}))$

end;

The following function checks if the histogram has a peak around the position $y$ :

- fun $\operatorname{Pn}(H, y)=$

let

fun $f$ y $x=\operatorname{Gauss}(x-y$, sigma);

val hist $=H * G p(\operatorname{Dom}(H), f(y), \operatorname{Size}(H))$

in

$\pi_{1, \max }$ (hist)

end;

The function returns a value between 0 and 1 representing the confidence that the histogram has a peak in the given position. A threshold can be applied if a hard decision is needed. With these functions, we can write the function MInv (H1, H2) that returns a number between 0 and 1 representing the confidence that a motion inversion takes place between the histograms $\mathrm{H} 1$ and $\mathrm{H} 2$ :

- fun $\operatorname{MInv}(H 1, H 2)=$

let

val $H=\operatorname{Sh}(P(H 1,1)+P(H 2,1))$;

val $\mathrm{y}=(\operatorname{dom}(H, 1) \cdot \mathrm{up}-\operatorname{dom}(H, 1) .10) / 2$;

in

$\operatorname{Pn}(H, y)$

end;

With this function, it is possible to formulate the query in the database as:

SELECT $r, s$

FROM MOTION

WHERE ABS $(r . t-$ s.t $)<=3 \operatorname{AND} \operatorname{MInv}(r . H, s . H)>0.5$ 


\section{SUMMARY AND OUTLOOK}

In this paper, we have argued that image database languages can be built based upon feature algebras and demonstrated how data manipulations and queries can be executed with a feature algebra in terms of histograms. While in this paper we have not formalized the exact query classes expressible in terms of this algebra, the peak detection and motion inversion examples, suggest that when embedded in a functional language, the algebra can express more complex queries than possible with current systems. We plan to investigate the expressiveness properties of histogram algebra in the future, and expect that it will provide us some insight into the desired properties of a more general feature algebra.

We envision two types in the advantages of feature algebras. The first is the flexibility that its operations allow in the description of the relevant similarity criteria for image databases. The relevant parts of the image features can be singled out at query time, using a high level algebra, rather than relying on the hard-coded operations typical of computer vision. In this sense, we note that the operations that we have defined for histograms apply to many different features which rely on similar data structures, e.g. Fourier transforms. The explicit extension of feature algebras to general image features is currently under way. The second advantage is that a description in terms of a feature algebra can bring many techniques developed for database (such as query optimization) and that, so far, have been difficult to extend to image and video data.

\section{Notes}

1. Some video operations such as joint color and motion-based object segmentation can provide features with both spatial and temporal support.

\section{References}

Abiteboul, S., Hull, R., and Vianu, V. (1995). Foundations of Dataases. Addison-Wesley Publishing Company, Inc., Reading, MA.

Adali, S., Bonatti, P., Sapino, M. L., and Subrahmanian, V. S. (1998). A multi-similarity algebra. In ACM SIGMOD International Conference on Management of Data, pages 402-413.

Day, Y., Wasfi, A.-K., R.Paul, and Ghafoor, A. (1998). Specification of a query language for multimedia database systems. In Proceedings International Workshop on Multimedia Software Engineering, pages 111-118. 
Fagin, R. (1999). Combining fuzzy information from multiple systems. Journal of Computer and System Science, 58:83-99.

Fagin, R. and Wimmers, E. L. (1999). A formula for incoporating weights into scoring rules. Theoretical Computer Science. Modified from "Incorporating user preferences in multimedia queries", in Proc. 6th International Conference on Database Theory, Delphi, Jan. 1997, SpringerVerlag Lecture Notes in Computer Science 1186, ed. F. Afrati and Ph. Kolaitis, 247-261.

Gupta, A. (1995). Visual information retrieval: a virage perspective. Technical report, Virage, Inc.

Hatipoglu, S., Mitra, S., and Kingsbury, N. (1999). Texture classification using dual-tree complex wavelet transform. In Proc. 7th International Congress on Image Processing and its Applications, pages 344-347.

Huang, J., Kumar, S. R., Mitra, M., Zhu, W.-J., and Zabih, R. (1997). Image indexing using color correlograms. In Proc. IEEE Computer Vision and Pattern Recognition Conference.

Li, J., Ozsu, M., Szafron, D., and Oria, V. (1997). MOQL: a multimedia object query language. In Third International Workshop on Multimedia Information Systems, pages 19-28.

Li, W., Candan, K., Hirata, K., and Hara, Y. (1998). Hierarchical image modeling for object based media retrieval. Data and Knowledge Engineering, 27:139-176.

Libkin, L., Machlin, R., and Wong, L. (1997). A query language for multidimensional arrays: Design, implementation, and optimization techniques. In ACM SIGMOD International Conference on Management of Data, pages 228-239.

Marathe, A. P. and Salem, K. (1997). A language for manipulating arrays. In VLDB 1997, International Conference on Very Large Data Bases, pages $46-55$.

Nepal, S. and Ramakrishna, M. (1999). Query processing issues in image (multimedia) databases. In Proc. 15th International Conference on Data Engineering, pages 22-29.

Santini, S. and Jain, R. (1999). Similarity measures. IEEE Transactions on Pattern Analysis and Machine Intelligence, 21(9).

Ullman, J. D. (1997). Elements of ML programming. Prentice-Hall.

\section{Biographies}

Amarnath Gupta Received a B.Tech. in Mechanical Engineering from the Indian Institute of Technology, Kharagpur in 1984, and an M.S. degree in Biomedical En- 
gineering from University of Texas at Arlington in 1987and Ph.D. (Engineering) in Computer Science from Jadavpur University India in 1994. He is an Assistant Research Scientist at the Center for Computational Science and Engineering, UCSD. Before joining UCSD he was a scientist at Virage, Inc, where he worked on multimedia information systems. Dr. Gupta's current research interests are in multimedia and spatiotemporal information systems, heterogeneous information integration and scientific databases.

Simone Santini Received the Laurea degree in Electrical Engineering from the Università di Firenze, Firenze (Italy) in 1990, the M.Sc. degree in Computer Science from the University of California, San Diego in 1996, and the Ph.D. Degree from the University of California, San Diego in 1998. From 1990 until 1994 Simone was with the Dipartimento di Sistemi e Informatica, Università di Firenze, and from 1998 to 1999 he was a project scientist at the Visual Computing Laboratory at the University of California, San Diego. He is currently visiting scientist at the Visual Computing Laboratory, UCSD, and scientist at Praja, Inc, where he workd on event detection, storage, and retrieval from multiple media streams. Simone's current research interests are image and video databases, event detection from multiple media streams, and spatiotemporal information systems. 